\title{
Factors affecting re-displacement in pediatric forearm fractures and the role of cast indices
}

\author{
Ender Alagöz, MD (D), Mehmet Akif Güleç, MD $\mathbb{D}$ \\ Department of Orthopedics and Traumatology, University of Health Sciences Bağcllar Training and Research Hospital, Istanbul, Turkey
}

Pediatric forearm fractures are among the most common reasons for presenting to the orthopedic emergency and pose challenges for orthopedists due to the complexity of their treatment and the frequency of complications. ${ }^{[1]}$ The majority of the childhood diaphyseal forearm fractures are treated with manipulative reductions and loss of reduction is one of the most commonly reported complications. ${ }^{[2,3]}$ The factors that cause loss of reduction can be categorized under three headings, related to the fracture, the surgeon or the patient. Fracture-based factors can be summarized as previous displacement, fracture localization, and the obliquity of the fracture, while surgeon-related factors are inadequate fracture reduction and poor casting technique, and patientrelated factors are muscle atrophy and the regression of the soft tissue edema in the cast. ${ }^{[2-4]}$ The part we need to focus on as trauma surgeons is the employment of an appropriate casting following a successful fracture reduction.

Although the importance of radiological indices in measuring the success of the treatment in distal radius fractures has been examined in many studies, studies on these indices in diaphyseal forearm fractures are very limited. Alemdaroğlu et al. ${ }^{[5]}$ described the three-point index (TPI) in adult and pediatric radius distal end fractures and reported

Received: September 27, 2019

Accepted: November 28, 2019

Published online: March 02, 2020

Correspondence: Ender Alagöz, MD. Bahçelievler Mah., Güneş 1 Sok., Metay B Blok, No: 9/16, 34180 Bahçelievler, İstanbul, Türkiye.

E-mail: enderalagoz@yahoo.com

Doi: $10.5606 /$ ehc. 2020.71523

Citation: Alagöz E, Güleç MA. Factors affecting re-displacement in pediatric forearm fractures and the role of cast indices. Jt Dis Relat Surg 2020;31(1):95-101.

\section{ABSTRACT}

Objectives: This study aims to investigate the factors affecting the loss of reduction in pediatric diaphyseal forearm fractures and to compare the three-point index (TPI) with the cast index, padding index, Canterbury index, and gap index.

Patients and methods: This retrospective study, which was conducted between January 2016 and December 2016, included 159 patients (134 males, 25 females; mean age 8.1 \pm 2.8 years; range, 3 to 13 years) with diaphyseal forearm fracture. Patients' age, gender, and the level, displacement, and location of the fractures were recorded. The presence of anatomic reduction, a straight ulnar border, and the cast type (banana or box type) were checked after the reduction. The TPI, cast index, padding index, Canterbury index, and gap index were measured.

Results: Fifty-two patients (32.7\%) experienced loss of reduction during the follow-up. Displaced fractures of both the radius and the ulna, cast type-banana, anatomical reduction, straight ulnar border, TPI, cast index, padding index, and Canterbury index were found to be associated with re-displacement.

Conclusion: Although cast indices can be used as a beneficial clinical tool in predicting the loss of reduction in the treatment of pediatric forearm fractures, they may not be sufficient when used alone. Obtaining a more accurate result may be possible by assessing several parameters such as the presence of an anatomic reduction, box-type casting, and TPI together.

Keywords: Casting technique, displacement, forearm fracture, pediatrics.

that the significance of the index in predicting the loss of reduction was higher than all other indices. ${ }^{[4]}$ They have attributed the success of this index to the basic three-point principle for achieving stability in the cast. The success of this index has been proven in many studies conducted subsequently. ${ }^{[6]}$ Iltar et al. ${ }^{[7]}$ modified the TPI for diaphyseal forearm fractures and found that this index was more successful in predicting the loss of reduction when compared to any other index. Therefore, in this study, we aimed to investigate the factors affecting the loss of reduction in pediatric diaphyseal forearm fractures and to 
compare the TPI with the cast index, padding index, Canterbury index, and gap index.

\section{PATIENTS AND METHODS}

Patients who applied to the emergency department of Bağcılar Training and Research Hospital due to diaphyseal forearm fracture between January 2016 and December 2016 were retrospectively investigated. The study included 159 patients (134 males, 25 females; mean age $8.1 \pm 2.8$ years; range, 3 to 13 years). Patients who had fractures that did not require reduction, had open, segmental fractures with dislocation (Monteggia, Galeazzi, etc.), had undergone an unacceptable reduction, had isolated radius or ulna fractures, concomitant fractures of the same extremity or a history of forearm fractures on the same side were excluded. In addition, as the reference points taken when calculating the TPI had to be $3 \mathrm{~cm}$ away from the joint and the fracture, fractures

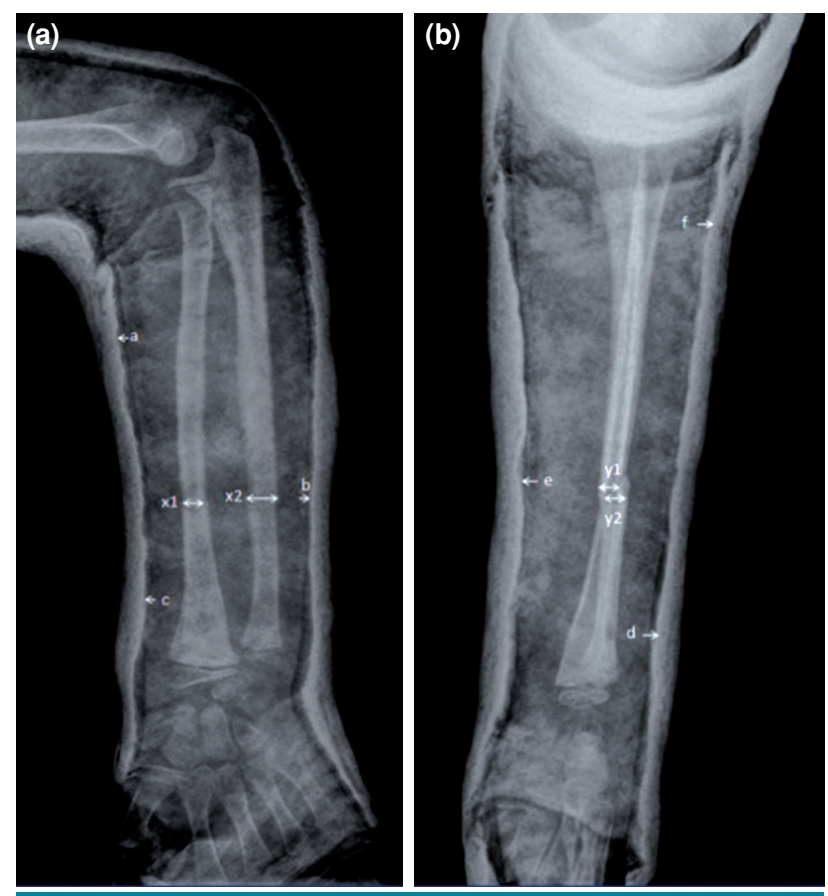

FIGURE 1. (a) Anteroposterior (AP) radiograph, showing measurement of proximal radial gap (a), fracture site ulnar gap (b) and radial gap (padding thickness) (c), sum of which was divided by sum of coronal reduced distance of radius (x1) and ulna (x2). (b) Lateral radiograph, showing measurement of distal dorsal gap (d), fracture site volar gap (e) and proximal radial gap ( $f$ ), sum of which was divided by sum of sagittal reduced distance of radius $(\mathrm{y} 1)$ and ulna (y2). Results of calculations of AP and lateral radiographs are added to find the three-point index $(a+b+c) /(x 1+x 2)+(d+e+f) /$ $(\mathrm{y} 1+\mathrm{y} 2)$. that did not meet this criterion were also excluded. None of the patients had neurovascular injury. The study protocol was approved by the Bağcilar Training and Research Hospital Ethics Committee. A written informed consent was obtained from parents of each patient. The study was conducted in accordance with the principles of the Declaration of Helsinki.

The first closed reduction and long arm casting were performed under sedation in the emergency department. Attention was paid to follow the general rules of casting while wrapping the long arm casts. Anteroposterior (AP) and lateral forearm radiographs were obtained before and after reduction. Acceptable reduction criteria for the patients were considered as an angulation of $<25^{\circ}$ for $<5$ years of age, $<20^{\circ}$ for five to nine years of age, and $<15^{\circ}$ for 10 years and older, based on the remodeling potential of the patients. The patients were called for weekly follow-ups after casting and AP and lateral forearm radiographs were taken.
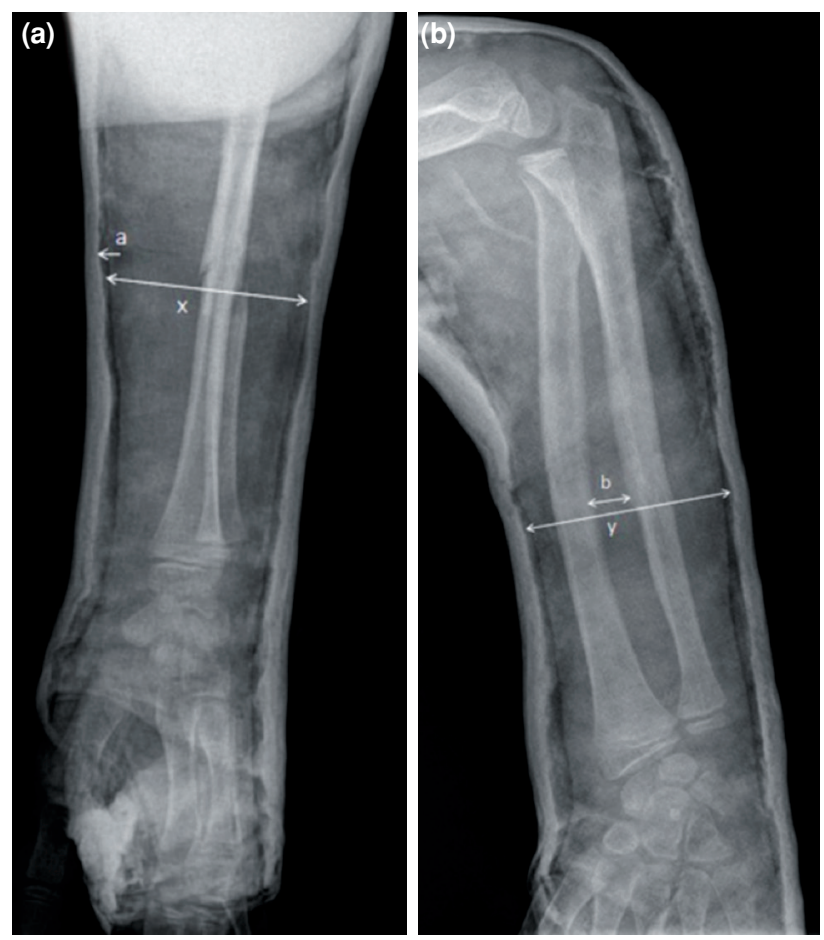

FIGURE 2. Padding index (a/b): division of padding thickness in plane of deformity on lateral radiograph (a) and maximum interosseous space in fracture site on anteroposterior (AP) radiograph. Cast index $(x / y)$ : division of internal cast width on lateral radiograph $(x)$ and internal cast width on $A P$ radiograph $(y)$. Canterbury index is sum of padding and cast indices. 
The files and radiological images of each patient were evaluated and their age and gender, whether the fractures were at the same or different levels (if the distance between two fractures $>2 \mathrm{~cm}$, they were accepted to be located at different levels), the initial displacement of each bone (displaced, greenstick, non-displaced), fracture locations (distal, middle, proximal), and the fracture apices (dorsal, volar) were recorded. Post-reduction radiographs were examined for the presence of anatomic reduction, a straight ulnar border, the cast type (banana or box type), and the TPI (Figure 1), cast index, padding index, Canterbury index (Figure 2), and gap index [(radial gap (fracture site) + ulnar gap (fracture site)/inner diameter of cast in AP plane) + (dorsal gap (fracture site) + volar gap (fracture site)/inner diameter of cast in lateral plane)] were measured as indicated in the literature. ${ }^{[2,7-9]}$ Iltar et al.'s

\begin{tabular}{|c|c|c|c|c|c|c|c|}
\hline & atien & nd fra & $\begin{array}{l}\text { ABLE I } \\
\text {-related fac }\end{array}$ & due & sss of & Iction & \\
\hline & & ss of $r$ & tion (-) & & s of $r e$ & tion (+) & \\
\hline & $\mathrm{n}$ & $\%$ & Mean \pm SD & $\mathrm{n}$ & $\%$ & Mean \pm SD & $p$ \\
\hline Age (year) & & & $8.4 \pm 2.8$ & & & $7.6 \pm 2.7$ & $0.077^{*}$ \\
\hline Gender & & & & & & & $0.190 \dagger$ \\
\hline Female & 14 & 13.1 & & 11 & 21.2 & & \\
\hline Male & 93 & 86.9 & & 41 & 78.8 & & \\
\hline Fracture location & & & & & & & $0.686 \dagger$ \\
\hline Different & 30 & 28.0 & & 13 & 25.0 & & \\
\hline Same & 77 & 72.0 & & 39 & 75.0 & & \\
\hline Fracture type-radius & & & & & & & \\
\hline Displaced & 62 & 57.9 & & 42 & 80.8 & & $0.005 \dagger$ \\
\hline Non-displaced & 5 & 4.7 & & 0 & 0.0 & & $0.173+$ \\
\hline Green stick & 40 & 37.4 & & 10 & 19.2 & & $0.021 \dagger$ \\
\hline Fracture type-ulna & & & & & & & \\
\hline Displaced & 55 & 51.4 & & 29 & 55.8 & & $0.605 \dagger$ \\
\hline Non-displaced & 12 & 11.2 & & 9 & 17.3 & & $0.287 \dagger$ \\
\hline Green stick & 40 & 37.4 & & 14 & 26.9 & & $0.191 \dagger$ \\
\hline Both bones displaced & & & & & & & $0.020 \dagger$ \\
\hline- & 70 & 65.4 & & 24 & 46.2 & & \\
\hline+ & 37 & 34.6 & & 28 & 53.8 & & \\
\hline Only radius displaced & & & & & & & $0.695 x$ \\
\hline- & 82 & 76.6 & & 38 & 73.1 & & \\
\hline+ & 25 & 23.4 & & 14 & 26.9 & & \\
\hline Only ulna displaced & & & & & & & $0.007 x$ \\
\hline- & 89 & 83.2 & & 51 & 98.1 & & \\
\hline+ & 18 & 16.8 & & 1 & 1.9 & & \\
\hline Apex & & & & & & & $0.120 \dagger$ \\
\hline Volar & 99 & 92.5 & & 44 & 84.6 & & \\
\hline Dorsal & 8 & 7.5 & & 8 & 15.4 & & \\
\hline Location-radius & & & & & & & \\
\hline Distal & 6 & 5.6 & & 8 & 15.4 & & $0.069 \dagger$ \\
\hline Mid & 90 & 84.1 & & 41 & 78.8 & & $0.413 \dagger$ \\
\hline Proximal & 11 & 10.3 & & 3 & 5.8 & & $0.346 \dagger$ \\
\hline Location-ulna & & & & & & & \\
\hline Distal & 24 & 22.4 & & 13 & 25.0 & & $0.719 \dagger$ \\
\hline Mid & 82 & 76.6 & & 39 & 75.0 & & $0.820 \dagger$ \\
\hline Proximal & 1 & 0.9 & & 0 & 0.0 & & $1.000 \dagger$ \\
\hline
\end{tabular}


modification was used when measuring the TPI. ${ }^{[7]}$ However, in the measurements conducted on the lateral radiographs, the point " $\mathrm{e}$ " was determined as the location of the fracture apex (dorsal or volar), taking the fracture apex and three-point reduction principle into account.

In the follow-up period, loss of reduction was considered in cases of an angulation of $>10^{\circ}$ in any direction in radial and/or ulna fractures. Follow-up radiographs were evaluated and losses of reduction were noted according to weeks.

\section{Statistical analysis}

The mean, standard deviation, median, minimum, maximum frequency and ratio values were used in descriptive statistics of the data. The distribution of the variables was measured by the KolmogorovSmirnov test. The Mann-Whitney U test was used for the analysis of quantitative independent data. The chi-square test was utilized in the analysis of qualitative independent data, and Fisher's test was used in cases the chi-square test conditions were not met. The effect level was investigated by univariate and multivariate logistic regression analysis. The Cox-regression (univariate/multivariate) and KaplanMeier tests were used for survival analysis. The results were evaluated at a significance level of $p<0.05$. The IBM SPSS version 22.0 software (IBM Corp., Armonk, NY, USA) was used in the analyses.

\section{RESULTS}

Different age groups were analyzed and no significant difference was found between the age groups in terms of loss of reduction. Demographic and fracture characteristics of the patients are given in Tables I and II. The female to male ratio was 1/5.4. Loss of reduction was observed in 52 (32.7\%) of the 159 patients during the follow-up. Losses of reduction in 21 patients $(40.4 \%)$ took place in the first week, in 25 patients (48.1\%) in the second week, and

\begin{tabular}{|c|c|c|c|c|c|}
\hline \multicolumn{6}{|c|}{$\begin{array}{c}\text { TABLE II } \\
\text { Comparison of reduction- and cast-related factors due to loss of reduction }\end{array}$} \\
\hline & \multicolumn{2}{|c|}{ Loss of reduction (-) } & \multicolumn{2}{|c|}{ Loss of reduction $(+)$} & \multirow[b]{2}{*}{$p$} \\
\hline & $\mathrm{n}$ & $\%$ & $\mathrm{n}$ & $\%$ & \\
\hline Anatomical reduction & & & & & $<0.001 \dagger$ \\
\hline- & 31 & 29.0 & 39 & 75.0 & \\
\hline+ & 76 & 71.0 & 13 & 25.0 & \\
\hline Cast type & & & & & $<0.001 \dagger$ \\
\hline Banana & 24 & 22.4 & 32 & 61.5 & \\
\hline Box & 83 & 77.6 & 20 & 38.5 & \\
\hline Straight ulnar border & & & & & $0.008 \dagger$ \\
\hline- & 48 & 44.9 & 35 & 67.3 & \\
\hline+ & 59 & 55.1 & 17 & 32.7 & \\
\hline Three-point index $(\geq 0.8)$ & & & & & $<0.001 \dagger$ \\
\hline- & 90 & 84.1 & 11 & 21.2 & \\
\hline+ & 17 & 15.9 & 41 & 78.8 & \\
\hline Cast index $(\geq 0.8)$ & & & & & $<0.001 \dagger$ \\
\hline- & 66 & 61.7 & 8 & 15.4 & \\
\hline+ & 41 & 38.3 & 44 & 84.6 & \\
\hline Padding index $(\geq 0.3)$ & & & & & $<0.001 \dagger$ \\
\hline- & 92 & 86.0 & 25 & 48.1 & \\
\hline+ & 15 & 14.0 & 27 & 51.9 & \\
\hline Canterbury index $(\geq 1.1)$ & & & & & $<0.001 \dagger$ \\
\hline- & 74 & 69.2 & 16 & 30.8 & \\
\hline+ & 33 & 30.8 & 36 & 69.2 & \\
\hline Gap index $(\geq 0.15)$ & & & & & $0.157 \dagger$ \\
\hline- & 85 & 79.4 & 36 & 69.2 & \\
\hline+ & 22 & 20.6 & 16 & 30.8 & \\
\hline
\end{tabular}




\begin{tabular}{|c|c|c|c|c|}
\hline Univariat & $\begin{array}{c}\text { TABLE } \\
\text { nultivariate analysi }\end{array}$ & sults for & isplacement & \\
\hline & Univariate model & & Iltivariate mo & \\
\hline & $p$ & OR & $95 \% \mathrm{Cl}$ & $p$ \\
\hline Radius-displaced & 0.006 & & & \\
\hline Radius-green stick & 0.023 & & & \\
\hline Both displaced & 0.022 & & & \\
\hline Anatomical reduction & $<0.001$ & 0.17 & $0.07-0.45$ & 0.000 \\
\hline Cast type-banana & $<0.001$ & 5.06 & $1.94-13.22$ & 0.001 \\
\hline Straight ulnar border & 0.009 & & & \\
\hline Three-point index $(<0.8)$ & $<0.001$ & 15.39 & $5.93-39.96$ & 0.000 \\
\hline Cast index $(<0.8)$ & $<0.001$ & 24.39 & 4.17-123.46 & 0.000 \\
\hline Padding index $(<0.3)$ & $<0.001$ & & & \\
\hline Canterbury index $(<1.1)$ & $<0.001$ & & & \\
\hline
\end{tabular}

in six patients $(11.5 \%)$ in the third week. No loss of reduction was observed after the third week.

Anatomic reduction was achieved in 89 patients $(56.0 \%)$ and $13(14.6 \%)$ of these patients experienced loss of reduction. Loss of reduction was observed in $39(55.7 \%)$ of the 70 patients $(44.0 \%)$ in which anatomic reduction could not be achieved $(\mathrm{p}<0.001)$. When banana- and box-type casts were compared, the loss of reduction in banana-type casts was significantly higher $(p<0.001)$. Similarly, patients with straight ulnar borders had significantly less loss of reduction $(p=0.008)$. Independent of ulnar fracture, loss of reduction was higher in displaced and greenstick fractures of the radius $(p=0.005$, $\mathrm{p}=0.021$, respectively). While patients with displaced fractures in both bones were found to be prone to loss of reduction $(\mathrm{p}=0.020)$, displaced fractures only in radius were not associated with loss of reduction $(\mathrm{p}=0.695)$. Interestingly, displaced fractures only in ulna showed a negative significance with loss of reduction $(\mathrm{p}=0.007)$.

When the radiological indices were evaluated, the TPI, cast index, padding index, and Canterbury index showed statistical differences between the groups $(\mathrm{p}<0.001$ in all). The gap index did not differ between the groups $(\mathrm{p}=0.157)$.

According to the multivariate logistic regression anatomical reduction, cast type, TPI, and cast index were associated with re-displacement $(p<0.001$, $p=0.001, p<0.001$, and $p<0.001$, respectively). Univariate and multivariate logistic regression analyses of the parameters with statistically significant differences are given in Table III. The sensitivity, specificity, positive, and negative predictive values of the radiological indices are given in Table IV.

\begin{tabular}{|c|c|c|c|c|c|}
\hline \multicolumn{6}{|c|}{ TABLE IV } \\
\hline & Sensitivity & Specificity & PPV & NPV & Accuracy \\
\hline & $\%$ & $\%$ & $\%$ & $\%$ & $\%$ \\
\hline Anatomical reduction & 75.0 & 71.0 & 55.7 & 85.4 & 72.3 \\
\hline Cast type-banana & 61.5 & 77.6 & 57.1 & 80.6 & 72.3 \\
\hline Three-point index & 78.9 & 84.1 & 70.7 & 89.1 & 82.4 \\
\hline Cast index & 84.6 & 61.7 & 51.8 & 89.2 & 69.2 \\
\hline Padding index & 51.9 & 86.0 & 64.3 & 78.6 & 74.8 \\
\hline Canterbury index & 69.2 & 69.2 & 52.2 & 82.2 & 69.2 \\
\hline Gap index & 30.8 & 79.4 & 42.1 & 70.2 & 63.5 \\
\hline
\end{tabular}




\section{DISCUSSION}

Acceptable restoration of the forearm functions can be achieved in most children following the non-operative treatment of diaphyseal forearm fractures. However, re-displacement is very common after this treatment, with a reported prevalence of up to $62 \% .{ }^{[10]}$ In our study, we found that $32.7 \%$ of all children had loss of reduction and all losses had occurred within the first three weeks.

The most important factors for a good plaster casting application are proper molding, a fine and uniform padding, and the three-point fixation. Poor casting technique is one of the most important factors affecting the loss of reduction. While distal forearm fractures are mostly treated with short arm casting, the multiplicity of the forces acting on the diaphyseal forearm fractures and the necessity of fixing the elbow joint make the treatment more complex. Distal slippage of the cast will lead to loss of reduction and can be minimized by ensuring that the arm is immobilized at a sharp right angle (box-type casting) and the ulnar border of the cast is kept straight..11] In our study, loss of reduction was significantly lower in the box-type casts and in the casts with straight ulnar borders $(p<0.001$ and $\mathrm{p}=0.008$, respectively).

Fracture-based and surgeon-related factors have been evaluated in several studies investigating the loss of reduction in forearm fractures. Complete displacement indicates severe injury to the periosteum and surrounding soft tissues and is associated with higher rates of re-displacement. ${ }^{[12,13]}$ Proctor et al.[12] identified two factors as the more important ones: an initial complete displacement and failure to achieve an anatomical reduction. In line with earlier studies, our results suggest that an anatomical primary reduction is protective against re-displacement, and displacement of both radius and ulna seems to be prone to re-displace during the follow-up. .7,12,13] $^{2}$ With regard to the side of fracture in relation to re-displacement, fractures in the proximal forearm have been considered particularly unstable and prone to re-displace, ${ }^{[14,15]}$ while we found that the side of fracture and different levels in those of both bones were not significant risk factors for re-displacement. There were no significant differences between the groups in terms of parameters such as age, gender, and the level of the fracture.

Plaster cast quality has historically been assessed in a subjective manner. No methods were available for objective analysis of the maintenance of reduction until Chess et al. ${ }^{[8]}$ developed the cast index, which was calculated on the basis of the cast geometry at the fracture site. The padding index and Canterbury index were designed by Bhatia and Housden ${ }^{[2]}$ to assess the cast technique for treatment of both diaphyseal and distal metaphyseal forearm fractures in children. The gap index was defined by Malviya et al. ${ }^{[9]}$ based on ratios of the gaps in the cast at the level of the fracture to the entire inside width of the cast in two planes. The TPI was first described by Alemdaroğlu et al. $^{[4]}$ for distal radius fractures and modified by Iltar et al. ${ }^{[7]}$ for pediatric forearm fractures. It differs from other indices because it not only takes into account the gaps at the fracture site, it is also based on physical principles and considers both three-point fixation in the cast and the accuracy of reduction. In their study, Iltar et al. ${ }^{[7]}$ compared the TPI with the cast, padding, and Canterbury indices and reported that TPI's sensitivity and specificity were higher than all other indices. In a recent study, Asadollahi et al. ${ }^{[10]}$ found that cast, padding, gap and three-point indices all have a strong correlation with re-displacement. In our study, we found that the cast indices other than the gap index were significant in predicting the loss of reduction. Although the accuracy of the TPI was higher than the other parameters, we concluded that no parameter alone could provide a definite prediction.

When multivariate logistic regression analysis of the parameters associated with re-displacement was performed after univariate logistic regression analysis, only anatomical reduction, casting type, the TPI, and cast index parameters were found to be associated with re-displacement $(p<0.001, p=0.001$, $\mathrm{p}<0.001$, and $\mathrm{p}<0.001$, respectively).

This study have some limitations. the first of these limitations is the retrospective nature of our study, and other is that all the reduction and casts were not performed by a single physician.

In conclusion, although cast indices can be used as a beneficial clinical tool in predicting the loss of reduction in the treatment of pediatric forearm fractures, they may not be sufficient when used alone. Although the accuracy of the TPI is slightly higher, it seems that the lack of assessment of the supracondylar molding is the weak aspect of the TPI. In our study, none of the patients who underwent an anatomic reduction had a box-type casting or a TPI of $<0.8$ had loss of reduction. Also, obtaining a more accurate result was possible by assessing several parameters such as the presence of an anatomic reduction, boxtype casting, and TPI together, while further studies are needed on this subject. 


\section{Declaration of conflicting interests}

The authors declared no conflicts of interest with respect to the authorship and/or publication of this article.

\section{Funding}

The authors received no financial support for the research and/or authorship of this article.

\section{REFERENCES}

1. Chung KC, Spilson SV. The frequency and epidemiology of hand and forearm fractures in the United States. J Hand Surg Am 2001;26:908-15.

2. Bhatia M, Housden PH. Re-displacement of paediatric forearm fractures: role of plaster moulding and padding. Injury 2006;37:259-68.

3. Ömeroğlu H. Basic principles of fracture treatment in children. Eklem Hastalik Cerrahisi 2018;29:52-7.

4. Alemdaroğlu KB, Iltar S, Cimen O, Uysal M, Alagöz E, Atlihan D. Risk factors in redisplacement of distal radial fractures in children. J Bone Joint Surg [Am] 2008;90:1224-30.

5. Alemdaroğlu KB, Iltar S, Aydoğan NH, Say F, Kilinç CY, Tiftikçi U. Three-point index in predicting redisplacement of extra-articular distal radial fractures in adults. Injury 2010;41:197-203.

6. Devalia KL, Asaad SS, Kakkar R. Risk of redisplacement after first successful reduction in paediatric distal radius fractures: sensitivity assessment of casting indices. J Pediatr Orthop B 2011;20:376-81.
7. Iltar S, Alemdaroğlu KB, Say F, Aydoğan NH. The value of the three-point index in predicting redisplacement of diaphyseal fractures of the forearm in children. Bone Joint $\mathrm{J}$ 2013;95-B:563-7.

8. Chess DG, Hyndman JC, Leahey JL, Brown DC, Sinclair AM. Short arm plaster cast for distal pediatric forearm fractures. J Pediatr Orthop 1994;14:211-3.

9. Malviya A, Tsintzas D, Mahawar K, Bache CE, Glithero PR. Gap index: a good predictor of failure of plaster cast in distal third radius fractures. J Pediatr Orthop B 2007;16:48-52.

10. Asadollahi S, Pourali M, Heidari K. Predictive factors for re-displacement in diaphyseal forearm fractures in children-role of radiographic indices. Acta Orthop 2017;88:101-8.

11. Herring JA, Ho C. Upper extremity injuries. In: Hering JA, editor. Tachdjian's pediatric orthopaedics. Dallas: Elsevier Saunders; 2014. p. 1333-40.

12. Proctor MT, Moore DJ, Paterson JM. Redisplacement after manipulation of distal radial fractures in children. J Bone Joint Surg [Br] 1993;75:453-4.

13. Yang JJ, Chang JH, Lin KY, Lin LC, Kuo CL. Redisplacement of diaphyseal fractures of the forearm after closed reduction in children: a retrospective analysis of risk factors. J Orthop Trauma 2012;26:110-6.

14. Bochang C, Jie Y, Zhigang W, Weigl D, Bar-On E, Katz K. Immobilisation of forearm fractures in children: extended versus flexed elbow. J Bone Joint Surg [Br] 2005;87:994-6.

15. Pretell Mazzini J, Rodriguez Martin J. Paediatric forearm and distal radius fractures: risk factors and re-displacement-role of casting indices. Int Orthop 2010;34:407-12. 